Combined atomic force microscope and electron-beam lithography used for the fabrication of variable-coupling quantum dots

M. C. Rogge, C. Fühner, U. F. Keyser, R. J. Haug, M. Bichler, G. Abstreiter, and W. Wegscheider

Citation: Appl. Phys. Lett. 83, 1163 (2003); doi: 10.1063/1.1599972

View online: https://doi.org/10.1063/1.1599972

View Table of Contents: http://aip.scitation.org/toc/apl/83/6

Published by the American Institute of Physics

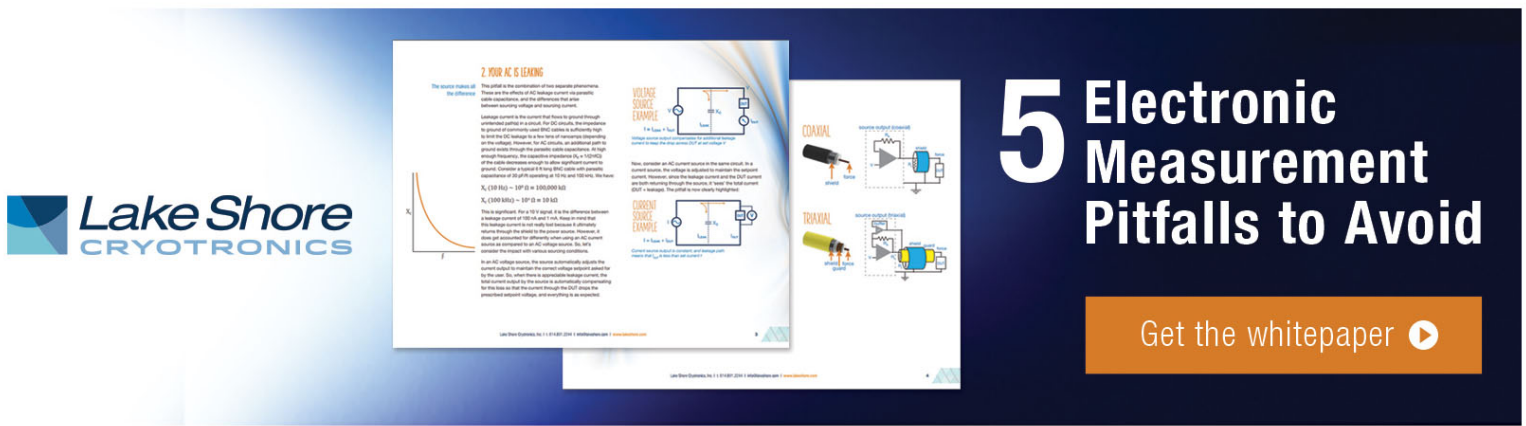




\title{
Combined atomic force microscope and electron-beam lithography used for the fabrication of variable-coupling quantum dots
}

\author{
M. C. Rogge, C. Fühner, ${ }^{a)}$ U. F. Keyser, and R. J. Haug \\ Institut für Festkörperphysik, Universität Hannover, 30167 Hannover, Germany \\ M. Bichler and G. Abstreiter \\ Walter Schottky Institut, TU München, 85748 Garching, Germany \\ W. Wegscheider \\ Angewandte und Experimentelle Physik, Universität Regensburg, 93040 Regensburg, Germany
}

(Received 31 March 2003; accepted 28 May 2003)

\begin{abstract}
We have combined direct nanofabrication by local anodic oxidation with conventional electron-beam lithography to produce a parallel double quantum dot based on a GaAs/AlGaAs heterostructure. The combination of both nanolithography methods allows fabrication of robust in-plane gates and $\mathrm{Cr} / \mathrm{Au}$ top-gate electrodes on the same device for optimal controllability. This is illustrated by the tunability of the interdot coupling in our device. We describe our fabrication and alignment scheme in detail and demonstrate the tunability in low-temperature transport measurements. (C) 2003 American Institute of Physics. [DOI: 10.1063/1.1599972]
\end{abstract}

Quantum dots (QDs) realized in various semiconductor materials have received great interest for the last decade. ${ }^{1}$ They are often called artificial atoms for the similarity of their zero-dimensional electronic spectra to their real counterparts. $^{2}$ In an expansion of this concept, two or more coupled QDs in close spatial vicinity form artificial molecules. ${ }^{3}$ QD molecules allow precise control of properties such as electron number, quantum mechanical state, or interdot coupling by experimental parameters. ${ }^{3-6}$ This tunability makes them promising candidates for the realization of quantum computers based on electron spin (see, e.g., Refs. 7-9). Recently, concrete steps towards a practical realization of this concept have been proposed. ${ }^{10}$

We focus on the fabrication of coupled QDs with both dots connected to common leads. With conventional electron-beam (e-beam) lithography, such devices were realized either with only one dot connected to the leads ${ }^{11}$ or with both dots connected to separate contacts. ${ }^{12,13}$ Only recently, Holleitner et al. fabricated parallel double QDs connected to common source and drain contacts by e-beam lithography. ${ }^{6,14}$ They used an additional e-beam patterned calixarene spacer layer ${ }^{15}$ to locally decrease the depletion created by top-gate electrodes in a two-dimensional electron system (2DES).

In our fabrication scheme, we combine conventional e-beam lithography and local anodic oxidation (LAO) of a 2DES using an atomic force microscope (AFM) ${ }^{16-18}$ LAO allows to pattern static insulating lines into the 2DES. These lines form the basic structure, including in-plane gates and tunneling barriers. LAO-based structures are easier to produce and operate, while being less sensitive to electrostatic discharges compared to generally more complex top-gate structures involving many gates. In addition, the potential walls created by LAO are extremely steep. ${ }^{19}$ We add a few metallic top-gate electrodes by e-beam lithography to improve the tunability of the basic structure. In our double QD

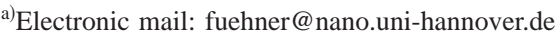

sample, a LAO-based tunneling barrier situated below a topgate electrode allows us to locally increase the depletion created by the gates. Thus, our combination of LAO and e-beam lithography allows us to enhance the tunability of LAObased structures without introducing the disadvantages and added complexity of equivalent purely top-gate-based structures.

In this letter, we present our nanofabrication scheme in detail. We demonstrate its feasibility by creation of a parallel QD molecule with tunable interdot coupling. Finally, we employ low-temperature transport measurements on this device.

An outline of our structure is shown in Fig. 1(a). Two LAO-based QDs are connected via 80-90-nm-wide point contacts to the source and drain reservoirs. In-plane gates 1 and 2 allow us to tune the electrochemical potential of the adjacent dot and tunneling barriers. The dots are connected by a 100-nm-wide opening to allow for tunnel coupling. The opening is covered by a top-gate electrode on the surface for electrostatic tuning of the interdot coupling. Due to the added depletion of the tunneling barrier underneath, the topgate can be operated at voltages that do not fully deplete the common 2DES drain contact.

Our fabrication scheme starts with a GaAs/AlGaAsheterostructure suitable for LAO. Our heterostructure contains a 2DES with a low-temperature mobility of $\mu$ $=42 \mathrm{~m}^{2} / \mathrm{V}$ s and a sheet density of $n=5 \times 10^{15} \mathrm{~m}^{-2}$ located $34 \mathrm{~nm}$ underneath the surface. The layer sequence consists of (from top to bottom): a 5-nm-thick GaAs cap layer, $8 \mathrm{~nm}$ of AlGaAs, the Si- $\delta$-doping, a 20 -nm-wide AlGaAs barrier, and $100 \mathrm{~nm}$ of GaAs. Using standard photolithography, we fabricate a mesa and $\mathrm{Au} / \mathrm{Ge} / \mathrm{Ni}$ ohmic contacts. Three nanolithographic steps, which have to be carefully aligned relative to each other, follow: (1) define a common coordinate system for the following steps by producing $\mathrm{Cr} / \mathrm{Au}$ markers using e-beam lithography, (2) LAO of the basic double-dot with in-plane gates, and (3) add the metallic finger gate with e-beam lithography. 


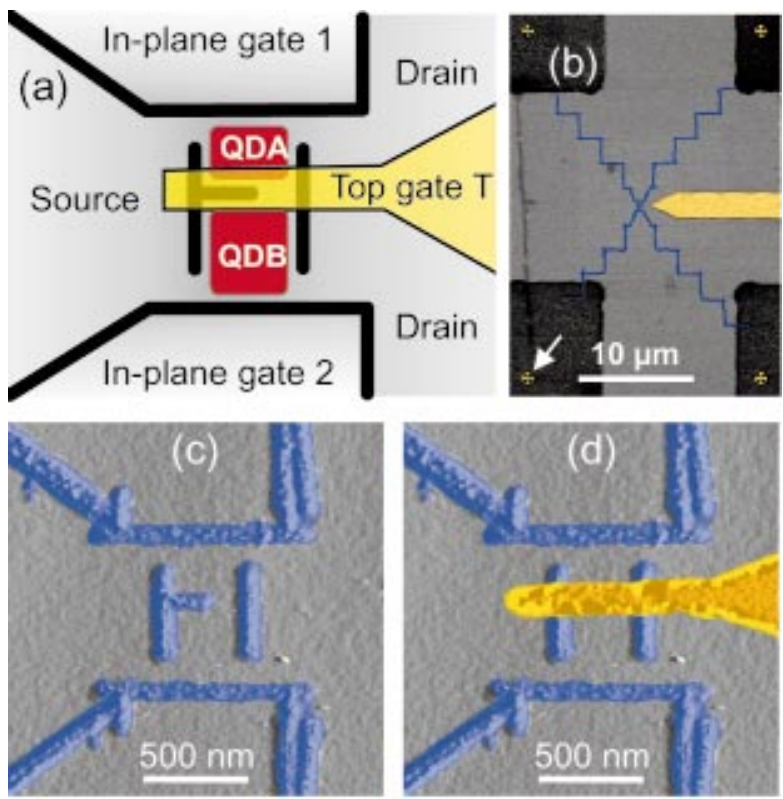

FIG. 1. (Color) (a) Schematic picture of our sample with two quantum dots QDA and QDB. The top gate controls the coupling of the dots without depleting the 2DES in the drain area. (b) Colorized scanning electron microscope picture after the local oxidation step (2) including mesa (gray), inner marker set (four yellow crosses, the one in the lower-left corner is highlighted by a white arrow), coarse part of the gate (yellow), and oxide lines (blue). The outer marker set is not shown. (c) Magnified colorized AFM image of the double-dot structure. (d) The same sample after adding the top gate (yellow) by e-beam lithography.

In the first step, we apply e-beam lithography to pattern a metal layer consisting of $7 \mathrm{~nm} \mathrm{Cr}$ and $30 \mathrm{~nm}$ Au for markers. Metallic alignment markers are needed to define a common e-beam/LAO coordinate system because the oxide lines produced by LAO are not visible in the electron microscope when covered by poly(methylmethacrylate) e-beam resist. We use two sets of markers: One set of markers is placed close to the center of the structure and is used to check the alignment of the later LAO step with the AFM, and the other one is placed farther outside where it is safely scanned with the electron microscope to align the final e-beam step without exposing the resist in the central dot region. In addition, we produce the coarse part of the gate that is used as an additional marker for LAO. This is shown in Fig. 1(b). The proximity of the LAO markers to the critical center of the structure allows us to align the later LAO step with a high absolute accuracy without suffering from distortions of the AFM piezo and a limited relative AFM resolution at large scanning fields.

For the AFM lithography, which is employed in the second nanolithography step, we use our LAO scheme described in Ref. 18. Local oxidation of the heterostructure surface locally modifies the band structure and depletes the 2DES underneath. This directly transfers the oxidized pattern from the surface into the electronic system. We fabricate the basic double-dot structure shown in Figs. 1(b) and 1(c). All oxide lines are about $100 \mathrm{~nm}$ wide and create insulating barriers in the 2DES. To align the AFM lithography, we use the coarse part of the gate produced in the previous e-beam step. After oxidation, we scan the LAO structure and the inner marker set with the AFM to check the alignment accuracy and to compensate for a possible error in the next step.
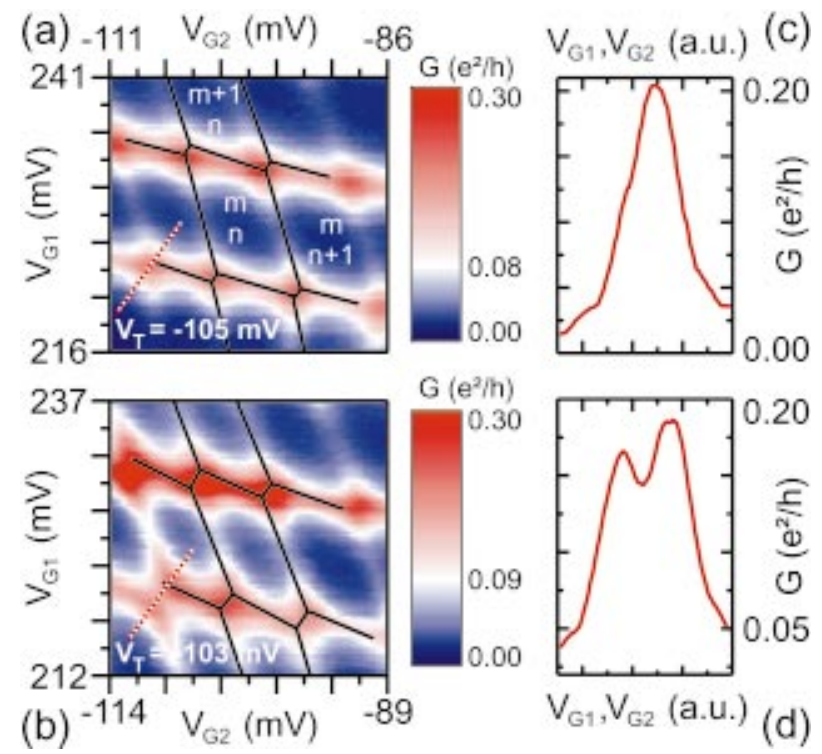

FIG. 2. (Color) (a) and (b) Gray-scale plot of the linear conductance $G$ as a function of in-plane gate voltages $V_{G 2}$ and $V_{G 1}$ for different interdot coupling strengths. In the hexagonal-shaped Coulomb blockade regions the electron number $(m, n)$ on dots $A$ and $B$ is stable. (c) and (d) Traces as marked in (a) and (b), respectively. The graphs clearly show the dependence of the Coulomb peak splitting on top-gate voltage.

We complete our sample by adding the 100-nm-wide gate finger ( $7 \mathrm{~nm} \mathrm{Cr} / 30 \mathrm{~nm} \mathrm{Au}$ ) using e-beam lithography. For alignment, we use the outer marker set from step (1).

The result is shown in Fig. 1(d). The figure illustrates how crucial an exact alignment of e-beam and AFM lithography relative to each other is. Since the width of the channel between the in-plane gates is $800 \mathrm{~nm}$, we need to control the vertical position of the finger gate with an accuracy better than $50 \mathrm{~nm}$. Only an exactly positioned finger gate allows us to influence mainly the interdot coupling instead of the tunneling barriers to the reservoirs. This accuracy is achieved with the scheme described earlier.

The tunable double QDs are investigated in transport measurements in a ${ }^{3} \mathrm{He} /{ }^{4} \mathrm{He}$-dilution refrigerator at a base temperature of $50 \mathrm{mK}$. We use a standard lock-in technique to measure the differential conductance with an ac-excitation voltage of $10 \mu \mathrm{V}$ at a frequency of $16 \mathrm{~Hz}$.

Figure 2(a) shows an image of the differential conductance $G$ as a function of in-plane gate voltages $V_{G 1}$ and $V_{G 2}$. Both dots are weakly coupled to the reservoirs. In the lowconductance regions, the electron number on $\operatorname{dot} A$ is $m$ and on $\operatorname{dot} B$ is $n .(m, n)$ is stable. The nearly horizontal lines
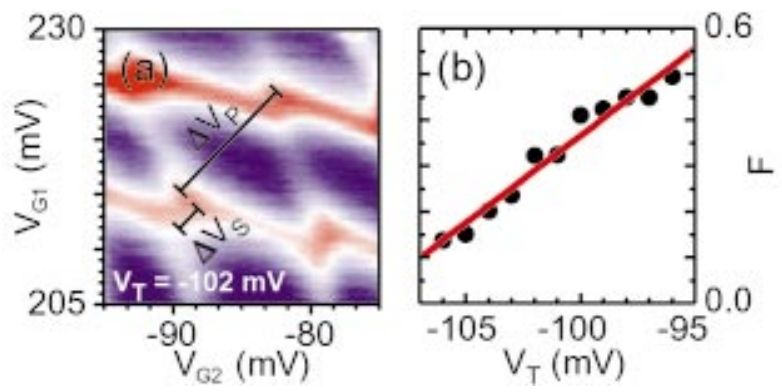

FIG. 3. (Color) (a) Peak spacing $\Delta V_{P}$ and peak splitting $\Delta V_{S}$ are determined from a gray-scale plot of the differential conductance $G$ as a function of gate voltages $V_{G 2}$ and $V_{G 1}$. (b) Fractional peak splitting $F$ $=2 \Delta V_{S} / \Delta V_{P}$ as a function of top-gate voltage $V_{T}$. 
denote changes of the charge on $\operatorname{dot} A$ by one electron; i.e., transitions from $(m, n)$ to $(m+1, n)$. The nearly vertical lines denote the respective transitions for dot $B$. At the intersections of both types of lines, both dots are in resonance with each other. This situation corresponds to transitions from $(m+1, n)$ to $(m, n+1)$. In Figure 2(a), each of these intersections is just split into two triple points, indicating a weak interdot coupling. ${ }^{3}$ We have increased the top-gate voltage from $V_{T}=-105 \mathrm{mV}$ to $-103 \mathrm{mV}$ in Fig. 2(b). The splitting of the triple points has clearly increased, leading to more obvious hexagonal Coulomb blockade domains. This indicates a stronger interdot coupling achieved by tuning the top gate voltage $V_{T}$. The traces in Figs. 2(c) and 2(d) illustrate the increase of the splitting of the triple points with increasing top-gate voltage [dashed lines in Figs. 2(a) and 2(b)].

The QDs are totally isolated from each other when we apply a top-gate voltage $V_{T}<-110 \mathrm{mV}$. We then get the signature of two noninteracting parallel QDs. To completely deplete the 2D electron gas in absence of an oxidized barrier a voltage of $V_{T}<-190 \mathrm{mV}$ is needed. So, there still is a common drain contact when the dots are isolated at $V_{T}=$ $-110 \mathrm{mV}$. At more positive top-gate voltages, the interdot coupling increases. At $V_{T}=0$, both dots have merged and we observe one single large QD. Thus, at intermediate voltages, the coupling of the two QDs is not purely capacitive, but at some point tunnel coupling begins.

The dependence of the interdot coupling on top-gate voltage is further investigated in Fig. 3. The coupling is characterized by the fractional peak splitting $F=2 \Delta V_{S} / \Delta V_{P}$ with $\Delta V_{S}$ the Coulomb peak splitting and $\Delta V_{P}$ the period. ${ }^{20}$ For totally decoupled dots $F$ is 0 , and for completely merged dots $F=1$. We observe a linear dependence of $F$ on $V_{T}$, ranging from $F=0.1$ at $V_{T}=-106 \mathrm{mV}$ to $F=0.55$ at $V_{T}=$ $-96 \mathrm{mV}$ in Fig. 3(b). This large range of fractional peak splitting observed demonstrates the tunability of the interdot coupling in our double-dot system nicely.

In conclusion, we have demonstrated the combination of local anodic oxidation with an AFM and electron-beam lithography on a GaAs/AlGaAs heterostructure. We have shown that this combination of well-known fabrication schemes allows an easy fabrication of advantageous gate layouts. We have fabricated two parallel quantum dots with tunable interdot coupling. The electronic properties of this device were investigated in transport measurements. We have demonstrated the tunability of the interdot coupling.

The authors thank F. Hohls and U. Zeitler for helpful discussions and help with the measurement setup. This work has been supported by BMBF.

${ }^{1}$ L. P. Kouwenhoven, C. M. Marcus, P. L. McEuen, S. Tarucha, R. M. Westerveld, and N. S. Wingreen, in Mesoscopic Electron Transport Series E, edited by L. L. Sohn, L. P. Kouwenhoven, and G. Schön (Kluwer, Dordrecht, 1997), Vol. 345, pp. 105-214.

${ }^{2}$ M. A. Kastner, Rev. Mod. Phys. 64, 849 (1992).

${ }^{3}$ W. G. van der Wiel, S. D. Franceschi, J. M. Elzerman, T. Fujisawa, S. Tarucha, and L. P. Kouwenhoven, Rev. Mod. Phys. 75, 1 (2003).

${ }^{4}$ L. P. Kouwenhoven, D. G. Austing, and S. Tarucha, Rep. Prog. Phys. 64, 701 (2001).

${ }^{5}$ R. H. Blick, D. Pfannkuche, R. J. Haug, K. v. Klitzing, and K. Eberl, Phys. Rev. Lett. 80, 4032 (1998).

${ }^{6}$ A. W. Holleitner, R. H. Blick, A. K. Hüttel, K. Eberl, and J. P. Kotthaus, Science 297, 70 (2002).

${ }^{7}$ D. Loss and D. P. DiVincenzo, Phys. Rev. A 57, 120 (1998).

${ }^{8}$ X. Hu and S. D. Sarma, Phys. Rev. A 61, 062301 (2000).

${ }^{9}$ V. N. Golovach and D. Loss, Semicond. Sci. Technol. 17, 355 (2002).

${ }^{10}$ L. M. K. Vandersypen, R. Hanson, L. H. W. van Beveren, J. M. Elzerman, J. S. Greidanus, S. D. Franceschi, and L. P. Kouwenhoven, in Quantum Computing and Quantum Bits in Mesoscopic Systems (Kluwer, Dordrecht, 2002).

${ }^{11}$ F. Hofmann, T. Heinzel, D. A. Wharam, J. P. Kotthaus, G. Böhm, W. Klein, G. Tränkle, and G. Weimann, Phys. Rev. B 51, 13872 (1995).

${ }^{12}$ L. W. Molenkamp, K. Flensberg, and M. Kemerink, Phys. Rev. Lett. 75, $4282(1995)$

${ }^{13}$ A. S. Adourian, C. Livermore, and R. M. Westervelt, Appl. Phys. Lett. 75, 424 (1999).

${ }^{14}$ A. W. Holleitner, C. R. Decker, H. Qin, K. Eberl, and R. H. Blick, Phys. Rev. Lett. 87, 256802 (2001).

${ }^{15}$ J. Fujita, Y. Ohnishi, Y. Ochiai, and S. Matsui, Appl. Phys. Lett. 68, 1297 (1996).

${ }^{16}$ M. Ishii and K. Matsumoto, Jpn. J. Appl. Phys. 34, 1329 (1995).

${ }^{17}$ R. Held, T. Vancura, T. Heinzel, K. Ensslin, M. Molland, and W. Wegscheider, Appl. Phys. Lett. 73, 262 (1998).

${ }^{18}$ U. F. Keyser, H. W. Schumacher, U. Zeitler, R. J. Haug, and K. Eberl, Appl. Phys. Lett. 76, 457 (2000).

${ }^{19}$ A. Fuhrer, S. Lüscher, T. Ihn, T. Heinzel, K. Ensslin, W. Wegscheider, and M. Bichler, Phys. Rev. B 63, 125309 (2001).

${ }^{20}$ J. M. Golden and B. I. Halperin, Phys. Rev. B 54, 16757 (1996). 